\title{
Diseño y análisis psicométrico de un instrumento que evalúa competencias básicas en Economía y Finanzas: una contribución a la educación para el consumo*
}

\author{
Design and Psychometric Analysis of an Instrument \\ Evaluating Basic Skills in Economics and Finance: \\ A Contribution to the Consumer Education
}

Recibido: septiembre 3 de 2012 | Revisado: marzo 6 de 2014 | Aceptado: mayo 17 de 2014

\author{
Mónica VARgas Prieto** \\ Fundación Universitaria Konrad Lorenz, Bogotá, Colombia \\ Bertha Lucía Avendaño Prieto *** \\ Fundación Universitaria Konrad Lorenz, Bogotá, Colombia
}

doi:10.11144/Javeriana.UPSY13-4.dapi

Para citar este artículo: Vargas, M., \& Avendaño, B. L. (2014). Diseño y análisis psicométrico de un instrumento que evalúa competencias básicas en Economía y Finanzas: una contribución a la educación para el consumo. Universitas Psychologica, 13(4), 1379-1393. http://dx.doi.org/10.11144/Javeriana.UPSY13-4.dapi

* Este artículo se deriva del trabajo de grado para optar por el título de Magister en Psicología del Consumidor de la Fundación Universitaria Konrad Lorenz

*** Contacto principal para correspondencia editorial. Correo electrónico: vmoni29@hotmail.com

**** Docente en la maestría de Psicología del Consumidor. Correo electrónico: blavendanop@yahoo.com
RESUMEN

En Colombia, se evidencia la falta de instrumentos válidos y confiables para realizar diagnósticos y mediciones que contribuyan a la definición de los contenidos de programas en educación económica y financiera y su efectividad en los objetivos que persiguen. El propósito del presente estudio fue diseñar y realizar el análisis psicométrico de un instrumento para evaluar competencias básicas en educación financiera y económica en estudiantes universitarios mayores de 15 años; se contó con la participación de 410 estudiantes. El instrumento se elaboró en concordancia con el plan de prueba, y la validación de contenido se realizó con ocho expertos. El estudio fue de tipo instrumental. Los datos se analizaron en el marco de la Teoría de Respuesta al Ítem (TRI). La consistencia interna establecida mediante el alfa de Cronbach arrojó un valor de 0.98. Los valores Infit y Outfit encontrados indicaron buen ajuste al modelo de Rasch. Los resultados permiten concluir que la prueba cumple con los requerimientos psicométricos exigidos para este tipo de instrumentos, constituyéndose en una herramienta útil para la medición de competencias básicas en economía y finanzas.

Palabras clave

educación para el consumo; educación económica; educación financiera; consumo responsable; competencias; TRI

\footnotetext{
A B S T R A C T

In Colombia the lack of valid and reliable instruments that allow diagnostics and measurements that contribute to the definition of the content of programs in economic and financial education and their effectiveness in their objectives is evident. The purpose of this study was to design and conduct a psychometric analysis of an instrument to assess basic skills in financial and economic education in 410 university students older than 15 . The instrument was developed according to the test plan and content validation was performed with 8 experts. The study was instrumental. Data were analyzed from the context of item response theory (IRT). Internal consistency was established by a Cronbach's alpha value of 0.98 . The values found for Outfit and Infit indicated good fit to the Rasch model. The results show that the test meets the psychometric requirements required for this type of instrument, becoming a useful tool for measuring basic skills in economics and finance. Keywords

consumer education; economic education; financial education; responsible consumption; competences; IRT
} 
El derecho a la educación para un consumo responsable surge con el objetivo de promover la libertad y la racionalidad en las prácticas consumistas (Ova1le, 2000, 2005). La reflexión sobre las implicaciones que el consumo tiene en la vida social de los individuos y el fuerte vínculo que existe entre consumo e identidad personal, resalta la importancia de una formación para el consumo responsable que tenga en cuenta tanto las características del consumidor (i. e., habilidad de autocontrol señalada por Sandoval, Caycedo, \& López, 2008) como las dinámicas del mercado. El consumidor debe ser capaz de evaluar activamente su relación con el mercado, para lo cual es necesario desarrollar habilidades y conocimientos particulares. Dentro de estas habilidades, autores como Compés (2002) destacan destrezas para investigar, asimilar y analizar críticamente la información de acuerdo con las necesidades individuales; evaluar el riesgo y ejercer juicios imparciales en la toma de decisiones responsables, comunicar efectivamente lo que puede pasar en las posibles situaciones de consumo y saber qué hacer o a dónde acudir cuando se presentan problemas específicos.

En diferentes estudios, se evidencia cómo el uso responsable de la información disponible mejora significativamente la eficiencia de la relación entre consumidor y mercado (Fast, Vosburgh, \& Frisbee, 1989; Haynes-Bordas, Kiss, \& Yilmazer, 2008; Peng, Bartholomae, Fox, \& Cravener, 2007). Fast et al. (1989) atribuyen la dificultad de tomar decisiones adecuadas de consumo al incremento significativo en la complejidad y número de bienes que ofrece el mercado, así como a las constantes innovaciones en los productos y las nuevas opciones sobre cómo y dónde pueden adquirirse. Esta idea va en concordancia con los resultados de varias investigaciones (Reifner \& Herwig, 2003) que muestran que como consecuencia de una cantidad de información excesiva, su selección y procesamiento se convierten en tareas complejas y consumidoras de tiempo para la mayoría de las personas (Loibl, Cho, Diekmann, $\&$ Batte, 2009). Se ha sugerido que la cantidad de información que el consumidor promedio es capaz de procesar es limitada (Reifner \& Herwig, 2003), condición que ha sido abordada en profundidad por diversos autores (Bettman, Luce, \& Payne, 1998;
Loibl et al., 2009), quienes concuerdan en que solo una pequeña parte de la información que rodea al producto es examinada cuidadosamente y usada en concordancia.

En contradicción con los argumentos que afirman que los consumidores más educados suelen implementar procesos extensivos en la búsqueda de información, autores como Erasmus, Boshoff y Rousseau (2001) señalan que, como resultado de su búsqueda, el consumidor no tomará decisiones racionales con base en esta, sino que basará su juicio en un heurístico de decisión, llevando a cabo una limitada aplicación de reglas, lo cual acarrea un sesgo a refugiarse en productos conocidos sobre los que ya tiene una idea previa y percibe como confiables. Lo anterior es importante si se tiene en cuenta que la educación para el consumo parte del principio de que todas las personas deben llegar a ser consumidores críticos y estar bien informadas acerca de los costos y la calidad de bienes y servicios que consumen, así como de los mecanismos, instancias y procedimientos para hacer valer sus derechos como consumidores. Los procesos educativos deben contemplar al consumidor como individuo y miembro de una comunidad, capaz de percibir el efecto de su conducta sobre el medio ambiente, ya que como lo señala Jarva (2011), en manos del consumidor promedio está el destino de la humanidad y del planeta.

La implementación de sistemas eficientes de consumo requiere niveles básicos de educación tanto financiera como global. Como se evidencia en las investigaciones de varios autores (Bitzer, 2005; Bitzer \& Troskie-deBruin, 2004; Crafford $\&$ Bitzer, 2009), bajos niveles educativos en áreas generales como la alfabetización, habilidades numéricas y comunicativas se asocian con problemas como la proliferación de tácticas abusivas de venta, adquisición indiscriminada de deudas, compra de productos defectuosos y recepción de servicios inadecuados por parte del consumidor. En este sentido, diversos autores (Hastings \& Tejeda-Ashton, 2008; Hilgert, Hogarth, \& Beverly, 2003; Lusardi $\&$ Tufano, 2009) han mostrado que mayores niveles de educación financiera están relacionados con decisiones más eficientes de consumo. Así mismo, 
estudios como los desarrollados por Bernheim, Garrett y Maki (2001) y Crafford y Bitzer (2009), por ejemplo, muestran que quienes han estado expuestos a la educación financiera son más propensos que otros a ahorrar, a planificar para su jubilación y, en general, como lo indican Lusardi y Mitchell (2008), a lograr una acumulación de mayores cantidades de riqueza. Adicionalmente, las acciones del consumidor afectan significativamente la dinámica del mercado nacional e internacional (Rezabakhsh, Bornemann, Hansen, \& Schrader, 2006).

Como respuesta a los diversos hallazgos que apuntan a la relación entre educación y eficiencia en el consumo, múltiples estudios (Atherton \& Wells, 1998; Crafford \& Bitzer, 2009; Rousseau, 1999) han propuesto la inclusión de programas de educación específica en esta área, con el fin de desarrollar competencias que disminuyan los posibles efectos adversos de su ausencia. Crafford y Bitzer (2009), por ejemplo, presentan una propuesta y una aplicación específica de este tipo de programas en el contexto universitario, planteando el desarrolo de currículos que contribuyan a cambiar las perspectivas y conductas de los estudiantes como actuales y futuros consumidores. La educación para el consumo en este sector de la población es indispensable dado que los jóvenes tienen una influencia enorme en el mercado global, ya sea mediante su papel de futuros empresarios (Ekanem \& Wyer, 2007), su conducta de compra compulsiva (Roberts \& Jones, 2001), sus hábitos de consumo, sus tendencias de crédito (Hayhoe, Leach, Turner, Bruin, \& Lawrence, 2000) e incluso sus respuestas a las campañas publicitarias (Wolburg, 2006).

A partir de la década de los sesenta, se comienzan a desarrollar programas enfocados a educar a los ciudadanos en economía y finanzas; algunos países pioneros en estos programas han sido Estados Unidos e Inglaterra a través de sus bancos centrales (Castro \& García, 2009). Los programas de Educación Económica y Financiera (EEF), persiguen alguno de estos tres objetivos: "(1) apoyar a los individuos para alcanzar sus metas personales, (2) mejorar el rendimiento del mercado financiero buscando que los consumidores cambien sus comportamientos, (3) contribuir al orden económico y social" (Gnan, Silgoner, \& Weber, 2007, pp. 28 32). En respuesta a esto, la educación financiera es reconocida a nivel mundial como un elemento fundamental de estabilidad y desarrollo económico y financiero (Organización para la Cooperación y el Desarrollo Económicos [OCDE], 2009). América Latina no ha sido ajena a estas tendencias mundiales, máxime cuando se evidencia la inequidad social, las brechas en la distribución de la riqueza y la falta de políticas que mejoren la calidad de vida de los ciudadanos. A pesar de este panorama, existen iniciativas desde los bancos centrales, el sector educativo, instituciones públicas y privadas y las asociaciones de consumidores, que permiten visualizar el fomento de políticas y el desarrollo de programas de EEF para los ciudadanos.

En Colombia, las primeras iniciativas se dan a finales de los años noventa (Castro \& García, 2009; García, 2012); sin embargo, cobran mayor fuerza en el país a raíz de la promulgación de la Ley 1328 de 2009, que establece los principios y reglas que regulan la relación entre el consumidor financiero y la entidades financieras, la cual hace énfasis en los derechos y protección del consumidor así como en los deberes que tienen las entidades financieras de promover y establecer programas de EEF y de garantizar la defensoría y protección del consumidor. Del Estatuto del Consumidor (Ley 1480 de 2011) que tiene como objetivo "proteger, promover y garantizar la efectividad y el libre ejercicio de los derechos de los consumidores, así como amparar el respeto a su dignidad y a sus intereses económicos" (Artículo 1..$^{\circ}$ y del planteamiento de una estrategia nacional de EEF, documento desarrollado en 2010 conjuntamente por el Ministerio de Hacienda y Crédito Público, el Ministerio de Educación Nacional, el Banco de la República, la Superintendencia Financiera de Colombia, el Fondo de Garantías de Instituciones Financieras, el Fondo de Garantías de Entidades Cooperativas y el Autorregulador del Mercado de Valores, que tiene como objetivo "dotar a los ciudadanos de los conocimientos y competencias básicas que les permitan ser sujetos activos dentro del sistema económico nacional, u optimizar su bienestar a través de la toma de mejores decisiones en materia financiera" (p. 17). 
Gnan et al. (2007) resaltan la relevancia de que todo programa enfocado a la EEF, parta de una línea base que determine las brechas en el conocimiento de los grupos objetivo. Al respecto, identifican al Test de Alfabetización Económica (Test of Economic Literacy [TEL]) de Estados Unidos, como una prueba estandarizada reconocida internacionalmente, que se utiliza para medir la compresión de conceptos económicos en estudiantes de secundaria y evaluar la efectividad de los planes de estudio. El TEL consta de 46 preguntas de selección múltiple que cubren cuatro categorías de conceptos: económicos fundamentales, microeconómicos, macroeconómicos y económicos internacionales. El TEL también ha sido adaptado en Alemania, Austria y Suiza y se utiliza para hacer comparaciones entre los mismos. Gnan y sus colaboradores revisaron diversos estudios que evidencian los siguientes resultados de la aplicación del TEL desarrollado por Soper y Walstad (1987), en el Nacional Council of Economic Education, de Estados Unidos: los estudiantes que toman cursos de economía obtienen resultados superiores en comparación con los estudiantes que no han tomados cursos; la alfabetización económica incrementa con la edad por la participación activa de las personas en la economía y los hombres obtienen desempeños superiores en comparación con los resultados de las mujeres. Sin embargo, el TEL ha recibido críticas porque las diferencias pueden ser resultado de la heterogeneidad de los sistemas educativos propios de cada país.

En Latinoamérica, a partir de distintos estudios sobre alfabetización económica, realizados en Chile, Caripán, Catalán y Hermosilla (2004 como se citó en Gempp et al., 2007), desarrollaron como instrumento de medición el Test de Alfabetización Económica para Adultos (TAE-A), inspirado en el TEL, el cual mide conceptos en cuatro áreas: Economía General, Microeconomía, Macroeconomía y Economía Internacional.

Gempp et al. (2007) trabajaron en abreviar el TAE-A a partir de la Teoría Clásica de los Test (TCT). El TAE-A inicialmente tenía 55 ítems y como resultado del análisis psicométrico definieron 25 ítems con distractores y claves apropiados en nivel de dificultad y capacidad de discriminación. La prueba también mostró una buena confiabili- dad $(\mathrm{GLB}=0.87)$ con niveles moderados de error de medida para todo el rango de sus puntuaciones observadas. Aunque los resultados obtenidos son prometedores en cuanto a validez, los autores afirman que aún son insuficientes para evidenciar la validez de constructo y la capacidad de los ítems para predecir comportamientos económicos cotidianos. Por lo tanto, recomiendan realizar más estudios psicométricos al TAE-A, con muestras aleatorias que permitan generalizar los resultados y calcular los errores de estimación.

En Barranquilla (Colombia), Amar, Abello, Denegri, Llanos y Suárez (2008) utilizaron el TAE para medir alfabetización económica en niños y adultos de estrato socioeconómico medio. Sin embargo, en investigaciones adelantadas en una institución de educación superior en Colombia, sugieren realizar ajustes al TAE para mejorar su validez de contenido (Amaya \& Sandoval, 2010; Naranjo \& Sandoval, 2010; Tavera, 2009), puesto que los análisis de contendido y de texto de los ítems evidencian que para aplicar el TAE en el contexto colombiano, es necesario realizar modificaciones en su lenguaje técnico y redacción.

En Colombia se han utilizado otros instrumentos direccionados por el Banco de la República para medir alfabetización económica y financiera o EEF. García (2012) realizó una investigación para medir el impacto a corto plazo del programa de "Finanzas para el Cambio" en 1.518 estudiantes de secundaria. Este programa, que inició en el 2006, tiene como propósito enseñar temas de economía básica y finanzas personales a estudiantes de educación básica secundaria de los grados noveno y décimo. Para medir el impacto, García utilizó la metodología Propensity Score Matching y diseñó una prueba con 27 ítems para evaluar aspectos básicos de economía y finanzas personales en cuanto a conocimientos, habilidades, actitudes, capacidades y comportamientos iníciales. De los 27 ítems, 13 fueron tomados de diferentes pruebas internacionales, y para su aplicación fueron traducidos y ajustados al contexto de la población objetivo. Los resultados del estudio evidencian que el programa de "Finanzas para el Cambio" favorece el nivel de conocimientos de los estudiantes en economía y finanzas personales "frente a estudiantes que 
no participan de programa pero reciben algún tipo de EEF en sus colegios. Sin embargo, el programa no logra cumplir con los objetivos que se propone en lo referente a lograr un efecto positivo en las habilidades, actitudes, capacidades y comportamientos de los estudiantes tratados, frente a los controles" (García, 2012, p. 32).

Otra iniciativa fue la encuesta de carga y educación financiera, contratada por el Banco de la República y aplicada por el Departamento Administrativo Nacional de Estadísticas (DANE) en el 2010 a 2.577 hogares en Bogotá que tienen algún producto financiero del sistema bancario (García, 2012). La encuesta tiene 10 ítems (Módulo de alfabetización financiera) que tienen el propósito de evaluar el nivel de competencias financieras y el uso de los servicios financieros. Los resultados evidencian que entre los jefes de hogar tienen mayor alfabetización financiera los hombres que las mujeres y que las personas mayores de 40 años tienen puntajes en aptitud financiera superiores a las de 18 a 40 años. Estos resultados son similares a los encontrados en pruebas internacionales, aunque las tasas de respuestas correctas son inferiores a las de países desarrollados (Ministerio de Hacienda y Crédito Público et al., 2010).

Aunque son varias las iniciativas y los esfuerzos que realizan diferentes instituciones en Colombia, todavía son limitadas las mediciones de los programas de EEF; es más sencillo medir cuando el objetivo del programa es evaluar el nivel de conocimientos, pero es complejo cuando se trata de modificar comportamientos como ahorrar, controlar el endeudamiento, entre otros. En el documento Estrategia Nacional de Educación Económica y Financiera (2010), se afirma que la mayoría de iniciativas en EEF ejecutan evaluaciones de satisfacción de los diferentes públicos objetivo, pero solo dos instituciones realizan medición de impacto de sus programas. Por lo tanto, resaltan la necesidad de establecer diagnósticos y realizar evaluaciones de impacto a los programas de EEF.

En este sentido, la OCDE (2013) está realizando una medición internacional en educación financiera, aplicable en todos los países. Parte de sus objetivos es proporcionar una guía detallada sobre el alcance y la definición operacional de la educación financiera, sin la necesidad de financiar estudios nacionales. Para tal fin, se desarrolló un instrumento, en el marco del Programa para la Evaluación Internacional de Alumnos (OCDE, 2013) que tiene como objetivo evaluar la preparación de los jóvenes para la vida, más allá de la escolaridad obligatoria y su capacidad para utilizar los conocimientos y habilidades financieras. La prueba de alfabetización financiera del programa PISA (por sus siglas en inglés), está compuesta por 40 ítems con un tiempo máximo de ejecución de 60 minutos; los ítems contemplan tres perspectivas: contenido, proceso y contexto, que tienen el propósito de medir el nivel de competencias de los jóvenes en este campo. Los primeros resultados de este estudio se conocerán en el 2014 y se espera que proporcionen datos que permitan comparar el rendimiento de los estudiantes dentro y entre los países y las economías participantes, que pueden ser usados por los Gobiernos para tomar decisiones basadas en la evidencia.

Los hallazgos considerados en este marco de referencia, manifiestan la falta de instrumentos válidos y confiables en el contexto colombiano que permitan realizar diagnósticos y mediciones, y proporcionen datos para la toma decisiones en cuanto a la definición de los contenidos de los programas de EEF y su efectividad en los objetivos que persiguen.

Teniendo en cuenta el anterior marco de referencia, el propósito de la investigación fue diseñar y realizar el análisis psicométrico de un instrumento para evaluar competencias básicas en economía y finanzas, en estudiantes mayores de 15 años.

\section{Método}

Se seleccionó una muestra estratificada con asignación proporcional por jornada. Participaron 410 estudiantes de primer y segundo semestre de una institución universitaria de Bogotá (237 de jornada diurna y 173 de nocturna), con edades comprendidas entre 16 y 39 años, un promedio de 21 años y una desviación estándar de 4 años. Para definir el tamaño de la muestra, se tuvo en cuenta: el tamaño de la población $N=1916$ (58 \% jornada diurna y $42 \%$ jornada nocturna), error 
$=5 \%$ y nivel de confianza $=97.7 \%$. El tipo de estudio fue instrumental, de acuerdo con la categorización de Montero y León (2007), en cuanto está encaminado al desarrollo de una prueba. Se diseñó una encuesta y una prueba (Vargas \& Avendaño, 2012). La encuesta para indagar sobre las variables sociodemográficas (género, edad, número de hijos, relación laboral, ingresos mensuales y estrato) y de comportamiento económico (ahorro, endeudamiento, uso de productos financieros y cursos relacionados con economía y finanzas). La prueba para evaluar competencias básicas en economía y finanzas incluye 25 ítems de selección múltiple con única y múltiple respuesta. La variable competencias básicas en economía y finanzas se conceptualizó como la capacidad que tiene el consumidor para identificar, interpretar, analizar y utilizar información económica y financiera en situaciones reales, con el fin de tomar decisiones libres y responsables. Para medir la variable se estableció el plan de prueba (Tabla 1), con dos criterios: el tipo de competencia y la información en economía y finanzas.

Se consultaron cinco expertos, profesionales en Economía, Finanzas, Contaduría Pública, Negocios Internacionales y Comercio Internacional, para definir las categorías y temas del plan de prueba (Tabla 1). Posteriormente, se elaboraron la encuesta y la prueba. Para la prueba se realizó la evaluación de contenido con ocho jueces. La encuesta y la prueba fueron sometidas a pilotaje y se aplicaron en línea. La participación de los estudiantes fue voluntaria y firmaron un consentimiento informado.

\section{Resultados}

Para alcanzar los objetivos propuestos, teniendo en cuenta el nivel de medición de cada una de las variables, se realizó el análisis de diferencias entre grupos y correlaciones entre los puntajes totales de la prueba y las variables sociodemográficas y de comportamiento económico; posteriormente, se realizó el análisis psicométrico del instrumento bajo el modelo de Rasch y se estableció el nivel de competencias básicas en economía y finanzas de los participantes.

\section{Análisis de diferencias entre grupos y correlaciones}

Inicialmente, se estableció la normalidad de la variable puntaje total, por medio de la prueba de Kolmogorov-Smirnov cuyo valor fue K-S $=1.5 \mathrm{con}$ $p=0.13$. Para comprobar si existían diferencias entre los grupos, se utilizó la prueba $t$ de Student para muestras independientes. Los resultados evidenciaron diferencias significativas entre las variables jornada, con un valor $t$ de -3.11 y un valor $p=0.001$ y la relación laboral con un valor $t=-3.33$ y $p=$ 0.001 frente al desempeño en la prueba; los estudiantes de la jornada nocturna, que en su mayoría se caracterizan por ser empleados, tienen promedios superiores en la prueba. Se encontraron diferencias estadísticamente significativas en las variables de comportamiento económico: presupuesto con un valor $t=-3.881$ y $p=0.001$, endeudamiento con un valor $t=-4.983$ y $p=0$ y tarjeta de crédito con $t=$

TABLA 1

Plan de prueba

\begin{tabular}{lcccccc}
\hline \multicolumn{1}{c}{ Competencia } & Macro-economía & Globalización & Finanzas & $\begin{array}{c}\text { Derechos del } \\
\text { Consumidor Financiero }\end{array}$ & $\begin{array}{c}\text { Total } \\
\text { ítems }\end{array}$ & $\%$ \\
\hline Solución de Problemas & 2 & 1 & 10 & 2 & 15 & 60 \\
Interpretativa & 5 & 1 & 2 & & 8 & 32 \\
Argumentativa & 2 & & & & 2 & 8 \\
Total ítems & 9 & 2 & 12 & 2 & 25 & 100 \\
$\%$ & 36 & 8 & 48 & 8 & 100 \\
\hline
\end{tabular}

Fuente: elaboración propia 
-2.65 y $p=0.008$; los puntajes más altos se encuentran en las personas que elaboran un presupuesto, tienen deudas y usan tarjeta de crédito (Tabla 2). Los datos también confirman diferencias significativas en los estudiantes que han tomado cursos de economía, $t=-3.030$ y $p=0.003$, microeconomía $t=-2.381$ у $p=0.019$, finanzas $t=-2.056$ у $p=$ 0.043 y presupuesto $t=-2.141$ y $p=0.035$, sus resultados son superiores frente a quienes no han tomado cursos relacionados.

Para comparar el puntaje total de la prueba con las variables estado civil, ingresos y estrato, se realizó un ANOVA, análisis de varianza de una vía (Tabla 3). Se encontraron diferencias estadísticamente significativas. El estrato 5 (nivel socioeconómico alto) presenta diferencias significativas en el promedio de la prueba en comparación con los estratos $3(p=0.006)$ y $4(p=0.015)$. El desempeño de los estudiantes de estrato 5 es superior. De igual manera, el promedio en el desempeño de la prueba es mayor en los estudiantes que tienen ingresos supe- riores a 5 salarios mínimos legales vigentes (SMLV) en comparación con quienes no tienen ingresos $(p$ $=0)$ o sus ingresos son inferiores a $4 \operatorname{SMLV}(p=$ $0, p=0$ y $p=0.019$, respectivamente). Respecto del estado civil, se encontró que los casados tienen desempeño superior en la prueba en comparación con los solteros $(p=0.002)$.

\section{Análisis con el modelo de Rasch}

Para realizar el análisis psicométrico, se utilizó el software Winsteps. En promedio, los estudiantes obtuvieron una puntuación de 10.7, las puntuaciones oscilaron entre 1 y 21 y ningún estudiante obtuvo la calificación máxima de 25 puntos. La medida se presenta en lógitos y fue igual a -0.36 , lo que indica una dificultad promedio. El valor outfit, estadígrafo de ajuste externo, fue de 1.02, indicando que en promedio las personas se ajustan a las expectativas del modelo. La correlación entre la puntuación natural de un individuo y su nivel

TABLA 2

Comparación de grupos mediante la Prueba t de Student

\begin{tabular}{|c|c|c|c|c|c|}
\hline Variables & Categoría & Media & Des. Est. & $\mathrm{t}$ - test & Sig. (bilateral) \\
\hline \multirow[t]{2}{*}{ Sexo } & Femenino & 10.56 & 3.29 & \multirow{2}{*}{-0.888} & \multirow{2}{*}{0.375} \\
\hline & Masculino & 10.85 & 3.47 & & \\
\hline \multirow[t]{2}{*}{ Jornada } & Diurna & 10.25 & 3.18 & \multirow{2}{*}{-3.117} & \multirow{2}{*}{$0.001 *$} \\
\hline & Nocturna & 11.31 & 3.54 & & \\
\hline \multirow[t]{2}{*}{ Relación laboral } & No trabaja & 10.33 & 3.34 & \multirow{2}{*}{-3.338} & \multirow{2}{*}{$0.001 *$} \\
\hline & Trabaja & 11.52 & 3.32 & & \\
\hline \multirow[t]{2}{*}{ Elabora Presupuesto } & No & 10.19 & 3.45 & \multirow{2}{*}{-3.881} & \multirow{2}{*}{$0.001 *$} \\
\hline & Sí & 11.48 & 3.13 & & \\
\hline \multirow[t]{2}{*}{ Ahorro } & No & 10.64 & 3.36 & \multirow{2}{*}{-1.405} & \multirow{2}{*}{0.166} \\
\hline & SÍ & 11.4 & 3.43 & & \\
\hline \multirow[t]{2}{*}{ Endeudamiento } & No & 10.1 & 3.3 & \multirow{2}{*}{-4.983} & \multirow{2}{*}{$0 *$} \\
\hline & Sí & 11.79 & 3.24 & & \\
\hline \multirow[t]{2}{*}{ Tarjeta de Crédito } & No & 10.44 & 3.32 & \multirow{2}{*}{-2.652} & \multirow{2}{*}{$0.008 *$} \\
\hline & SÍ & 11.45 & 3.42 & & \\
\hline
\end{tabular}

*Diferencias significativas al nivel de 0.01 .

Fuente: elaboración propia 
TABLA 3

Comparación de grupos mediante ANOVA con un factor

\begin{tabular}{|c|c|c|c|c|c|}
\hline Variables y Categorías & Media & $\mathrm{DE}$ & (I)Categoría & (J) Categoría & Sig. (bilateral) \\
\hline \multicolumn{6}{|l|}{ Estrato } \\
\hline 1 & 11.63 & 2.72 & 5 & 1 & 0,583 \\
\hline 2 & 11.04 & 3.14 & & 2 & 0.087 \\
\hline 3 & 10.36 & 3.34 & & 3 & $0.006 * *$ \\
\hline 4 & 10.47 & 3.53 & & 4 & $0.015 *$ \\
\hline 5 & 12.38 & 3.51 & & 6 & 0.9 \\
\hline 6 & 12.23 & 3.52 & & & \\
\hline \multicolumn{6}{|l|}{ Estado civil } \\
\hline Casado & 13.19 & 4.1 & Casado & Separado & 0.617 \\
\hline Separado & 12.25 & 2.22 & & Soltero & $0.002 * *$ \\
\hline Soltero & 10.58 & 3.31 & & Unión libre & 0.058 \\
\hline Unión libre & 10.94 & 3.55 & & & \\
\hline \multicolumn{6}{|l|}{ Ingresos mensuales } \\
\hline Ninguno & 9.85 & 3.38 & Más de 5SMLV & Ninguno & $0 * *$ \\
\hline Menos de $1 \mathrm{SMLV}$ & 10.75 & 3.18 & & Menos de $1 \mathrm{SMLV}$ & $0 * *$ \\
\hline $1 \mathrm{SMLV}$ a $2 \mathrm{SMLV}$ & 11.01 & 2.97 & & $1 S M L V$ a $2 S M L V$ & $0 * *$ \\
\hline 2SMLV a 3SMLV & 12.09 & 3.07 & & 2SMLV a 3SMLV & $0.019 *$ \\
\hline 3SMLV a 4SMLV & 10.43 & 3.32 & & 3SMLV a 4SMLV & $0.001 * *$ \\
\hline 4SMLV a 5SMLV & 13.14 & 3.29 & & 4SMLV a 5SMLV & 0.323 \\
\hline Más de 5SMLV & 14.67 & 4.19 & & & \\
\hline
\end{tabular}

*La diferencia de medias es significativa al nivel 0.05 .

** La diferencia de medias es significativa al nivel 0.01.

Fuente: elaboración propia

de habilidad calculado es de 0.99 , lo cual indica estimaciones adecuadas del parámetro $b$ o parámetro de dificultad de los individuos. El valor para el $\alpha$ de Cronbach de los 25 ítems fue de 0.98, lo que señala alta consistencia interna de la prueba. El nivel de ajuste para los puntajes infit MNSQ es de 1, valor que muestra ajuste perfecto del ítem según la expectativa del modelo. El valor ZSTD es de 0.06, lo cual es consistente con el MNSQ de 1 . El valor outfit fue de 1.02 que indica que, en promedio, los ítems se encuentran en una situación óptima para el proceso de medición.

La Tabla 4 presenta los estadísticos de ajuste de los ítems al modelo de Rasch, bajo los títulos infit (ajuste interno) y outfit (ajuste externo), cuyas medias cuadráticas o MNSQ tienen una expectativa de 1 , con un umbral de aceptabilidad entre 0.8 y 1.3 . Para el ajuste interno, todos los valores de MNSQ caen dentro del umbral de aceptabilidad; en cuanto a los valores del ajuste externo, dos de los 25 ítems (denominados Derechos 2 y Ahorro 3) sobrepasan el umbral de aceptabilidad de 1.3, lo cual sugiere algunas respuestas al azar de individuos que no poseían la habilidad estimada para acertarlos o de quienes se esperaba que contestaran correctamente, no lo hicieron. El valor $\theta$ para cada uno de los ítems, indica la posición relativa de dificultad en la escala logit que va de $-\infty$ a $+\infty$, de tal manera que a mayor valor de $\theta$ más difícil es el ítem. Los ítems más difíciles fueron los denominados Derechos 2 y Desempleo, que hacen referencia a los derechos del consumidor financiero y a la toma de decisiones en un escenario de desempleo, respectivamente. Los ítems más fáciles fueron los denominados Presupuesto e Inflación 1, los cuales se refieren al manejo del presupuesto y a la comprensión del concepto de inflación, respectivamente. 
TABLA 4

Estadísticos de ajuste al modelo de Rasch para los ítems

\begin{tabular}{lcccccc}
\hline & & & \multicolumn{2}{c}{ INFIT } & \multicolumn{2}{c}{ OUTFIT } \\
\cline { 5 - 7 } Ítem & $\theta$ & $\begin{array}{c}\text { Error St del } \\
\text { modelo }\end{array}$ & MNSQ & ZSTD & MNSQ & ZSTD \\
\hline Derechos 2 & 1.47 & 0.14 & 1.13 & 1.5 & 1.33 & 2.4 \\
Desempleo & 1.25 & 0.13 & 1.06 & 0.7 & 1.15 & 1.3 \\
Intereses 1 & 1.06 & 0.12 & 1.03 & 0.5 & 1.07 & 0.8 \\
Tarjeta 1 & 0.91 & 0.12 & 0.97 & -0.5 & 1.01 & 0.2 \\
Ahorro 2 & 0.84 & 0.12 & 1.04 & 0.6 & 1.04 & 0.5 \\
Inflación 2 & 0.69 & 0.11 & 1.02 & 0.4 & 1.01 & 0.1 \\
Uso Crédito & 0.68 & 0.11 & 1 & 0.1 & 0.96 & -0.5 \\
Ahorro 3 & 0.59 & 0.11 & 1.09 & 1.8 & 1.38 & 5 \\
Política & 0.51 & 0.11 & 0.95 & -1.1 & 0.93 & -1.1 \\
Instrumentos & 0.3 & 0.11 & 1.11 & 2.8 & 1.14 & 2.4 \\
Derechos 1 & 0.2 & 0.11 & 1.04 & 1.2 & 1.04 & 0.8 \\
Ahorro 4 & 0.07 & 0.11 & 0.95 & -1.6 & 0.94 & -1.3 \\
Inversión & -0.13 & 0.1 & 0.97 & -0.8 & 0.96 & -1 \\
Deflación & -0.18 & 0.1 & 0.98 & -0.6 & 0.98 & -0.6 \\
Tarjeta 2 & -0.19 & 0.1 & 1.04 & 1.4 & 1.04 & 1.1 \\
Ahorro 1 & -0.24 & 0.1 & 0.98 & -0.7 & 0.96 & -1.1 \\
PIB 1 & -0.32 & 0.1 & 0.99 & -0.5 & 1 & -0.1 \\
Intereses 2 & -0.36 & 0.1 & 0.89 & -3.8 & 0.89 & -3.1 \\
Devaluación & -0.68 & 0.11 & 0.95 & -1.7 & 0.93 & -1.7 \\
PIB 2 & -0.71 & 0.11 & 0.89 & -3.3 & 0.88 & -2.9 \\
IPC & -0.8 & 0.11 & 1 & -0.1 & 1.01 & 0.2 \\
TLC & -1.03 & 0.11 & 0.9 & -2.4 & 0.9 & -1.9 \\
Tipo de crédito & -1.19 & 0.11 & 1.01 & 0.3 & 0.97 & -0.5 \\
Presupuesto & -1.30 & 0.11 & 1.04 & 0.8 & 1.09 & 1.3 \\
Inflación 1 & -1.42 & 0.12 & 0.93 & -1.2 & 0.88 & -1.7 \\
\hline
\end{tabular}

Fuente: elaboración propia

La Figura 1 muestra la alineación de los ítems y la habilidad de los estudiantes organizados por grupos de habilidad: la primera columna presenta los puntajes logit, que para el caso indica que los 25 ítems de la prueba se distribuyeron entre -1.42 y 1.47 logit.

\section{Análisis del nivel de competencias}

Para analizar el nivel de competencias, se clasificó la variable puntaje total de la prueba en cinco rangos construidos a partir de la media (10.7) y la deviación estándar (3.37). El nivel de competencia obtenido en la prueba se discriminó por la variable jornada
(Tabla 5). Contiene la participación de las variables edad, ingresos y relación laboral, de tal forma que se puede caracterizar a los estudiantes de la jornada diurna, en su mayoría, en un rango de edad de 16 a 21 años, que no tienen trabajo y que no reciben o tienen ingresos inferiores a un SMLV. Los estudiantes de la jornada nocturna se caracterizan por ser mayores de 21 años, trabajan y tienen ingresos entre uno y cuatro SMLV. Del análisis se observa que el $51 \%$ del total de estudiantes de la jornada diurna se encuentra en un nivel "bajo" de competencias básicas en economía y finanzas, seguido por el 39 \% que se encuentra en un nivel "medio" y un $11 \%$ en nivel "alto". En contraste, los estudiantes de la jornada nocturna quedaron clasificados en su 


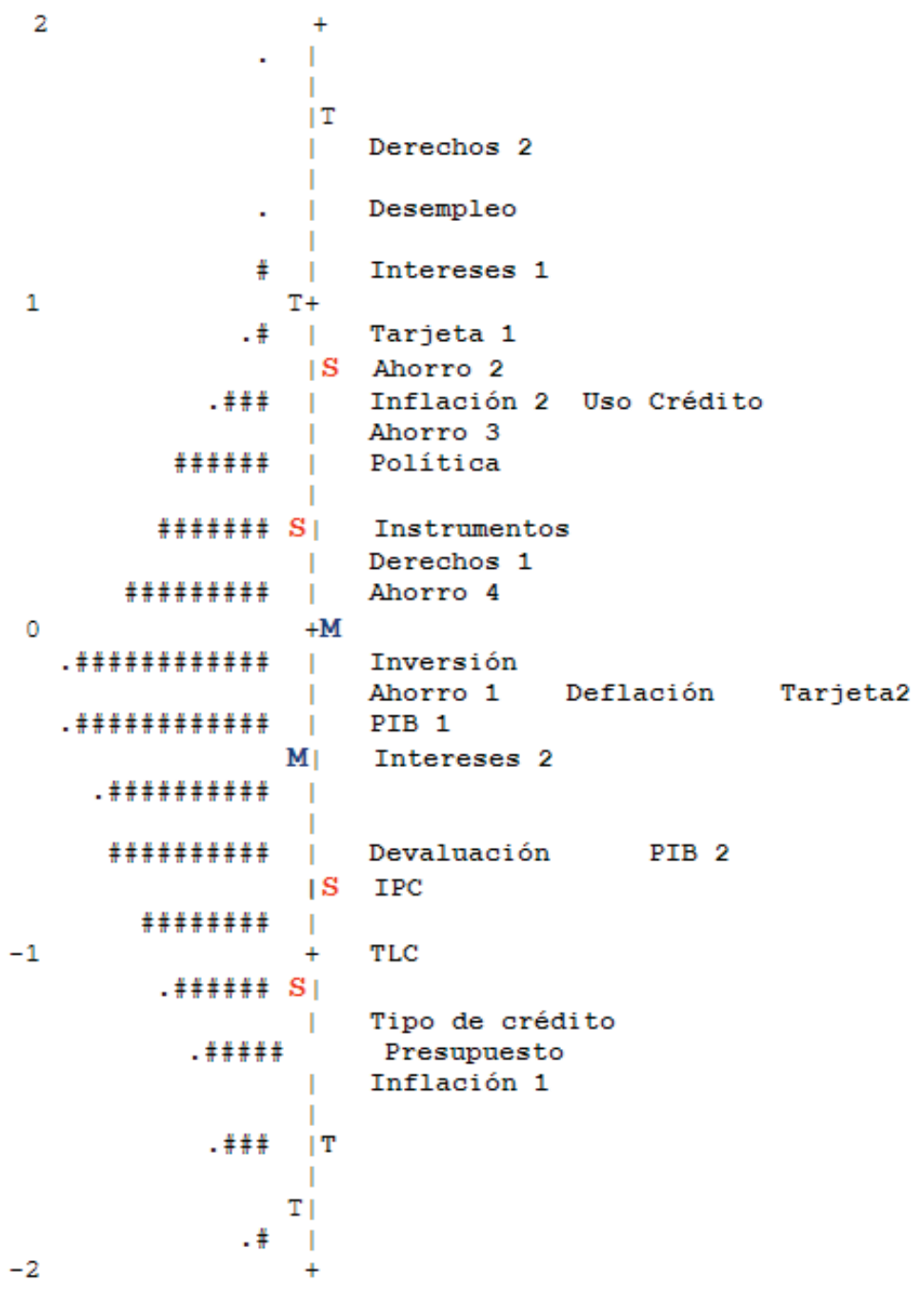

Figura 1. Calibración de los participantes y de los ítems a lo largo de la variable.

Fuente: elaboración propia

mayoría (59\%), en niveles “medio" y "alto”, el 40 \% de estudiantes de esta jornada tienen "bajo" nivel de competencias.

\section{Discusión}

Los resultados del estudio arrojan información valiosa en educación económica y financiera, principalmente en tres aspectos: (1) permiten contrastar los hallazgos encontrados en diferentes investigaciones; (2) aportan un instrumento cuyos ítems se encuentran en situación óptima para el proceso de medición y (3) brinda un diagnóstico del nivel de competencias básicas en los estudiantes evaluados.

El análisis de resultados evidencia que en el puntaje total obtenido en la prueba, no existen diferencias estadísticamente significativas entre los estudiantes hombres y mujeres. Estos resultados no concuerdan con los hallazgos de Walstad y Robson (1997), quienes indican que los hombres tienen resultados superiores en las pruebas de economía; los autores aclaran que estas diferencias son explicadas por las influencias sociales y culturales que crean estereotipos de roles sexuales y tienden a disminuir el interés de las mujeres por temas de 
TABLA 5

Clasificación del nivel de competencia por jornada

\begin{tabular}{lcccccc}
\hline \multirow{2}{*}{ Nivel de Competencia } & \multicolumn{2}{c}{ Diurna } & \multicolumn{2}{c}{ Nocturna } & Total & Total \\
& $n$ & $\%$ & $n$ & $\%$ & $n$ & $\%$ \\
\hline Muy bajo & 50 & 21 & 24 & 14 & 74 & 18 \\
Bajo & 70 & 30 & 45 & 26 & 115 & 28 \\
Medio & 92 & 38 & 75 & 43 & 167 & 41 \\
Alto & 23 & 10 & 23 & 13 & 46 & 11 \\
Muy alto & 2 & 1 & 6 & 3 & 8 & 2 \\
\hline Total & 237 & 100 & 173 & 100 & 410 & 100 \\
\hline
\end{tabular}

Fuente: elaboración propia

economía que tradicionalmente se consideran dominados por los hombres; diferencias cognitivas en razonamiento matemático y espacial y diferencias en las instrucciones dadas por los maestros. Lo mismo sucede con los resultados que arrojó la encuesta de carga y educación financiera (Ministerio de Hacienda y Crédito Público et al., 2010), que indica que los hombres jefes de hogar obtuvieron resultados superiores en alfabetización financiera en comparación con las mujeres jefe de hogar. Al respecto una posible explicación es la presentada por Gempp et al. (2007), quienes sugieren que las diferencias de género no tienen un valor explicativo en la alfabetización económica, si se analiza aislada de otros factores como el nivel de escolaridad.

La variable relación laboral permitió diferenciar a los estudiantes que no trabajan de los que trabajan, estos últimos obtuvieron resultados superiores en la prueba. Adicionalmente, se encontró que a mayor edad el desempeño es superior. Estos hallazgos concuerdan con los resultados de la revisión que efectúan Gnan et al. (2007), quienes reportan que la alfabetización económica básica aumenta con la edad, independientemente de la escolaridad y que seguramente esto se debe a la participación activa en la economía. Así mismo, se encontraron diferencias significativas en las variables de tipo sociodemográfico: estrato, estado civil y nivel de ingresos. En concordancia con los hallazgos de García (2012), García, Acosta y Rueda (2013) y Llanos, Denegri, Amar, Abello y Tirado (2009), los estudiantes con mayor nivel de ingresos obtuvieron un desempeño superior en la prueba. Las anteriores deducciones justifican la necesidad que tiene Colombia de definir y aprobar políticas nacionales que fomenten la educación para el consumo dirigidas a los diferentes actores de la sociedad.

El resultado más importante del presente estudio fue la elaboración de un instrumento para evaluar competencias básicas en economía y finanzas; la revisión teórica evidenció instrumentos eficientes para medir conocimiento en estas áreas, pero débiles en su alcance para medir las competencias, con el propósito de fomentar la toma de decisiones conducentes a un consumo responsable. Según la valoración de los expertos, el instrumento incluye los temas básicos que debe evaluar (validez de contenido) y es prometedor en su validez convergente al corroborar varios de los hallazgos descritos anteriormente.

Los resultados presentados sugieren que la evaluación de competencias en economía y finanzas posiblemente incluye elementos que normalmente no son abordados en cursos enfocados o relacionados con estas áreas. Esta es una posible explicación de los resultados obtenidos en la evaluación que se realizó a los participantes. Aunque se encontró que los estudiantes de la jornada nocturna tienen mayores niveles de competencia, sus promedios de ejecución indican que es necesario trabajar en las competencias que requieren; no es suficiente comprender los conceptos económicos y financieros, sino tener la capacidad para interpretar, analizar y utilizar la información de los productos y servicios financieros y exigir mayor calidad; tener un conocimiento preciso de los derechos y deberes como 
consumidor financiero, es vital para mantener el equilibrio en la economía, de esta forma, será un agente activo dentro de la misma y facilitará la toma de decisiones.

Un consumidor competente en economía y finanzas estará en la capacidad de comprender qué es la economía y cómo opera el sistema económico en el que interactúa. Tendrá las habilidades para identificar, interpretar y analizar información referente a un producto o servicio financiero. Su nivel de competencias le permitirá tomar decisiones, reflexionando conscientemente sobre algunos cuestionamientos como: ¿Qué es lo que tengo (presupuesto)?, ¿Cómo funciona el producto financiero que voy a adquirir?, ¿Cuál producto selecciono en medio de tantos nuevos y complejos?, ¿Por qué necesito ese producto?, ¿Qué derechos, obligaciones y costos tengo sobre ese producto?, ¿Cuáles son las consecuencias de su consumo? La educación para el consumo no debería dejar de suministrar información, sino complementarla con el desarrollo de habilidades dirigidas a interactuar con el mercado de una forma eficiente. Como afirman Loibl et al. (2009), el acceso a buena información sobre cómo invertir el dinero para la jubilación (iqué casa comprar o la forma de financiarla?) puede ser poco útil si los consumidores carecen de la confianza y las habilidades personales necesarias para establecer una relación equitativa con el mercado.

Consumidores informados y educados para el consumo potencializan la toma de decisiones y elecciones conducentes a un consumo responsable que promueva un mejoramiento en su calidad de vida (Sandoval et al., 2008). La educación para el consumo se muestra como un elemento capaz de contribuir al desarrollo de estrategias en los individuos para establecer una relación menos asimétrica con el mercado y más equitativa entre los elementos constitutivos de una comunidad, a la vez que potencializa la estabilidad económica y financiera, en tanto, como señalan autores como Lusardi y Mitchell (2008), consumidores educados financieramente toman decisiones más informadas, pero también exigen servicios de mayor calidad, fomentando la competencia y la innovación en el mercado.
Finalmente, los resultados indican que los programas de educación económica y financiera son necesarios para todo ciudadano; estas competencias deben incorporarse desde la niñez a través de las instituciones educativas (escuelas, colegios), para ir fomentando la cultura de ahorro y la comprensión del sistema económico que opera en nuestro país. El impacto de los programas debe ser valorado a través de instrumentos válidos y confiables.

\section{Limitaciones y posibles aplicaciones}

Son múltiples los aspectos que afectan la relación de consumo; el desempeño eficaz del consumidor en la toma de decisiones va más allá del acceso a cierto conocimiento financiero que le permita guiar su comportamiento, incluye además un conocimiento del sí mismo y un reconocimiento real de sus necesidades.

El instrumento analizado en el presente estudio constituye una herramienta útil para la identificación del nivel de competencias básicas en economía y finanzas en consumidores universitarios. Sin embargo, evaluar las competencias que requiere todo ciudadano en esas materias, no es una tarea fácil, dada la complejidad y amplitud de los temas que se deben evaluar. Por lo tanto, se sugiere profundizar en cada una de las categorías evaluadas y continuar realizando investigaciones, con dos finalidades esenciales: ampliar su ámbito de aplicación a otros grupos de edad, con el fin de cubrir poblaciones menores de 15 años o mayores de 40 años, ya que también son consumidores, y realizar aplicaciones que permitan obtener información útil para que todas aquellas personas que participan en la formación y educación de niños y adolescentes, puedan identificar y encausar sus competencias, a través de diversas metodologías, reformas curriculares y cambios en la infraestructura tanto personal como física.

Un aspecto que llama la atención es que pocos autores han investigado explícitamente los efectos de los niveles limitados de transparencia del mercado y los costos de búsqueda de información en la posición de poder de los consumidores. Investigaciones al respecto serían de alta relevancia 
ya que involucran la toma de decisiones. También se recomienda incluir en estudios futuros, de manera más exhaustiva, una categoría que evalúe el conocimiento y competencias sobre los derechos y deberes del consumidor financiero, ya que muchas decisiones no acertadas las toma el consumidor por su falta de conocimiento de la normatividad que garantiza el cumplimento de sus deberes y el ejercicio de sus derechos.

\section{Referencias}

Amar, J., Abellos, R., Denegri, M., Llanos, M., \& Suárez, R. (2008). Estrategias de socialización económica en padres de familia. Revista Económica del Caribe, 1, 156-183.

Amaya, G., \& Sandoval, M. (2010). Análisis de contenido y ajuste del test de alfabetización económica para adultos (TAE-A) en una muestra de adultos de la ciudad de Bogotá (Tesis de maestría inédita). Fundación Universitaria Konrad Lorenz, Bogotá, Colombia.

Atherton, M., \& Wells, J. (1998). Consumer education: Learning for life. Consumer Policy Review, 8(4), 127-131.

Bernheim, D., Garrett, D., \& Maki, D. (2001). Education and saving: The long-term effects of high school financial curriculum mandates. Journal of Public Economics, 85, 435-565.

Bettman, J. R., Luce, F. M., \& Payne, J. W. (1998). Constructive Consumer Choice Processes. Journal of Consumer Research, 25(3), 187-217.

Bitzer, E. M. (2005). First-year students' perceptions of generic skills competence and academic performance: A case study at one university. South African Journal of Higher Education, 19(3), 172-187.

Bitzer, E. M., \& Troskie-de Bruin, C. (2004). The effect of factors related to prior schooling on student persistence in higher education. South African Journal of Education, 24(2), 119-125.

Castro, F., \& García, N. (2009). La educación económica y financiera en los bancos centrales de América Latina. Revista del Banco de la República, 83(984), 4-33.

Colombia, Ley 1328 de 2009. Por la cual se dictan normas en materia financiera, de seguros, de mercado de valores y otras disposiciones. Julio 15 de 2009, DO. №47411.

Colombia, Ley 1480 de 2011. Por medio de la cual se expide el Estatuto del Consumidor y se dictan otras disposiciones. Octubre 12 de 2011, DO. №48220.

Compés, R. (2002). Atributos de confianza, normas y certificación: comparación de estándares para hortalizas. Economía Agraria y Recursos Naturales, $1,115-130$.

Crafford, S., \& Bitzer, E. M. (2009). Consumer learning for university students: A case for a curriculum. Higher Education Research and Development, 28(4), 443-455.

Departamento Administrativo Nacional de Estadística. (2010). Informe preliminar de Resultados. Recuperado de http://www.dane.gov.co/files/investigaciones/boletines/carga_fin/Informe_semestral_preliminar_Encuesta_Financiera.pdf

Ekanem, I., \& Wyer, P. (2007). A fresh start and the learning experience of ethnic minority entrepreneurs. International Journal of Consumer Studies, 31(2), 144-151.

Erasmus, A., Boshoff, E., \& Rousseau, G. (2001). Consumer decision-making models within the discipline of consumer science: A critical approach. Journal of Family Ecology and Consumer Sciences, 29, 72-81.

Fast, J., Vosburgh, R., \& Frisbee, W. (1989). The effects of consumer education on consumer search. Journal of Consumer Affairs, 23(1), 65-90.

García, N. (2012). El impacto de la educación económica y financiera en los jóvenes: el caso de finanzas para el cambio. Borradores de Economía, 687, 2-47.

García, N., Acosta, F., \& Rueda, J. (2013). Determinantes de la alfabetización financiera de la población bogotana bancarizada. Borradores de Economía, 792, 1-24.

Gnan, E., Silgoner, M., \& Weber, B. (2007). Economic and financial education: Concepts, goals and measurement. Monetary Policy $\mathbb{E}$ the Economy, 3, 28-49.

Gempp, R., Denegri, M., Caripán, N., Catalán, V., Hermosilla, S., \& Caprile, C. (2007). Desarrollo del Test de Alfabetización Económica para adultos TAE-A-25. Interamerican Journal of Psychology, 41(3), 275-284. 
Hastings, J., \& Tejeda-Ashton, L. (2008, diciembre). Financial literacy, information, and demand elasticity: Survey and experimental evidence from Mexico (NBER: Papel de trabajo № 14538). Cambridge, MA: National Bureau of Economic Research.

Hayhoe, C., Leach, L., Turner, P., Bruin, M., \& Lawrence, F. (2000). Differences in spending habits and credit use of college students. Journal of Consumer Affairs, 34(1), 113-133.

Haynes-Bordas, R., Kiss, D. E., \& Yilmazer, T. (2008). Effectiveness of financial education on financial management behavior and account usage: Evidence from a 'Second Chance' Program. Journal of Family and Economic Issues, 29(3), 362-390.

Hilgert, M., Hogarth, J., \& Beverly, S. (2003). Household financial management: The connection between knowledge and behavior. Federal Reserve Bulletin, 89(7), 309-322.

Jarva, V. (2011). Consumer education and everyday futures work. Futures, 43(1), 99-111.

Llanos, M., Denegri, M., Amar, J., Abello, R., \& Tirado, D. (2009). Aprendiendo a comprender el mundo económico. Barranquilla, CO: Ediciones Uninorte.

Loibl, C., Cho, S., Diekmann, F., \& Batte, M. (2009). Consumer self-confidence in searching for information. The Journal of Consumer Affairs, 43(1), 26-55.

Lusardi, A., \& Mitchell, O. (2008). Planning and financial literacy: How do women fare? American Economic Review, 98(2), 413-417.

Lusardi, A., \& Tufano, P. (2009). Teach Workers about the Perils ofDebt. Harvard Business Review, 22-24.

Ministerio de Hacienda y Crédito Público, Ministerio de Educación Nacional, Banco de la República, Superintendencia Financiera de Colombia, Fondo de Garantías de Instituciones Financieras, Fondo de Garantías de Entidades Cooperativas \& Autorregulador del Mercado de Valores (2010). Estrategia nacional de educación económica y financiera. Una propuesta para su implementación en Colombia. Bogotá: Autores.

Montero, I., \& León, O. (2007). A guide for naming research studies in Psychology. International Journal of Clinical and Health Psychology, 7(3), 847-862.

Naranjo, J., \& Sandoval, M. (2010). Análisis de la comprensión que poseen las y los estudiantes de 4 a
11 grado de edades entre los 7 y los 17 años, de la ciudad de Bogotá, de las preguntas y las opciones de respuesta del test de alfabetización económica (TAE-N). (Tesis de maestría inédita). Fundación Universitaria Konrad Lorenz, Bogotá, Colombia.

Organización para la Cooperación y el Desarrollo Económicos. (2009). Proyecto de la OECD sobre educación financiera y su red internacional de educación financiera. Recuperado de http://www.oecd.org/ finance/financial-markets/43138294.pdf

Organización para la Cooperación y el Desarrollo Económicos. (2012). PISA 2012. Finalcial Literacy Framework. Recuperado de http://www.oecd.org/pisa/ pisaproducts/PISA\%202012\%20framework\%20 e-book_final.pdf

Ovalle, J. (2000). Derechos del consumidor. México: Universidad Nacional Autónoma de México/Cámara de Diputados, LVII Legislatura.

Ovalle, J. (2005). Los derechos de los consumidores. Revista de Derecho Privado, Nueva Época, 4(12), 75-111.

Peng, T. -C. M., Bartholomae, S., Fox, J. J., \& Cravener, G. (2007). The impact of personal finance education delivered in high school and college courses. Journal of Family and Economic Issues, 28, 265-284. DOI: 10.1007/s10834-007-9058-7

Reifner, U., \& Herwig, I. (2003). Consumer education and information rights in financial services. Information 8 Communication Technology Law, 12(2), 125-142.

Rezabakhsh, Bornemann, Hansen \& Schrader (2006). Consumer Power: A Comparison of the Old Economy and the Internet Economy. Journal of Consumer Policy, 29:3-36.

Roberts, J. A., \& Jones, E. (2001). Money attitudes, credit card use and compulsive buying among American college students. Journal of Consumer Affairs, 35(21), 213-240.

Rousseau, D. (1999). Consumerism and social responsibility. En P. J. Du Plessis \& G.G. Rousseau (Eds.), Buyer behaviour: A multi-cultural approach (pp. 373-394). Johannesburg: Thomson.

Sandoval, M., Caycedo, C., \& López, W. (2008). El consumo inteligente más allá del libre albedrío: una visión desde el autocontrol. Formación Continuada 
a Distancia. Consejo General de Colegios Oficiales de Psicólogos, 3-27.

Soper, J.C., \& Walstad, W.B. (1987). Test of Economic Literacy: Examiner's Manual (2nd ed.). New York: Joint Council on Economic Education.

Tavera, C. (2009). Estado de la alfabetización económica en colegios de estrato socioeconómico 3, 4 y 5 de la ciudad de Bogotá. (Tesis de maestría inédita). Fundación Universitaria Konrad Lorenz, Bogotá, Colombia.

Vargas, M., \& Avendaño, B. L. (2012). Diseño y análisis psicométrico de un instrumento que evalúa competencias básicas en economía y finanzas: una contribución a la educación para el consumo. (Tesis de maestría inédita). Fundación Universitaria Konrad Lorenz, Bogotá, Colombia.

Walstad, W., \& Robson, A. (1997). Differential item functioning and male-female differences on multiple-choice tests in economics. The Journal of Economic Education, 28(2), 155-171.

Wolburg, J. M. (2006). College students' responses to anti-smoking messages: Denial, defiance and other boomerang effects. Journal of Consumer Affairs, 40(2), 294-323. 
